\title{
Canadian Journal of Microbiology
}

\section{Toxoplasma gondii infection induces the formation of host's nuclear granules containing poly (A)-binding proteins}

\begin{tabular}{|c|c|}
\hline Journal: & Canadian Journal of Microbiology \\
\hline Manuscript ID & cjm-2017-0755.R1 \\
\hline Manuscript Type: & Article \\
\hline Date Submitted by the Author: & 29-Jan-2018 \\
\hline Complete List of Authors: & $\begin{array}{l}\text { Fischer, Karlee; University of Windsor, Chemistry and Biochemistry } \\
\text { Roberts, Mikayla; University of Windsor, Chemistry and Biochemistry } \\
\text { Roscoe, Scott; University of Windsor, Chemistry and Biochemistry } \\
\text { Avci, Yasin; University of Windsor, Chemistry and Biochemistry } \\
\text { Ananvoranich, Sirinart; University of Windsor, Chemistry and Biochemistry }\end{array}$ \\
\hline $\begin{array}{r}\text { Is the invited manuscript for } \\
\text { consideration in a Special } \\
\text { Issue? : }\end{array}$ & N/A \\
\hline Keyword: & $\begin{array}{l}\text { Host-parasite interaction, Nuclear granules, mRNP, Stress response, } \\
\text { Toxoplasma }\end{array}$ \\
\hline
\end{tabular}


1 Toxoplasma gondii infection induces the formation of host's nuclear granules containing poly

\section{2 (A)-binding proteins}

4 Karlee Fischer ${ }^{1}$, Mikayla Roberts ${ }^{1}$, Scott Roscoe, Yasin Avci, and Sirinart Ananvoranich*

6 Department of Chemistry and Biochemistry, University of Windsor, Windsor, Ontario, Canada, $7 \quad \mathrm{~N} 9 \mathrm{~B} 3 \mathrm{P} 4$

$11{ }^{1}$ Both authors equally contributed.

12

$13 \quad *$ To whom correspondence should be addressed:

14 Sirinart Ananvoranich, Department of Chemistry and Biochemistry, University of Windsor, 401

15 Sunset Avenue, Windsor, Ontario, Canada, N9B3P4, Tel: (519) 253-3000 ext 3550, Fax: (519) 973-

16 7098, E-mail: anans@uwindsor.ca

20 Running title: nuclear granules formed upon Toxoplasma infection 


\section{Abstract}

To study a mechanism by which human host cells respond to an infection of Toxoplasma

27 gondii, we monitored the level of poly-(A) binding protein (PABP), an indicator of translation. Here

28 we report an observation of the relocalization of PABPs in human host cells upon $T$. gondii

29 infection. Notably the aggregates of PABPs formed upon infection are mainly found in the nucleus,

30 which is different from the response found in an exposure to a heat shock. The treatment of the

31 infected monolayers with pyrimethamine inhibits the multiplicity of the parasite, and reverses the

32 relocalization of PABP aggregates. This active interaction between the infected mammalian host

33 cells and $T$. gondii appears to be different than that caused by viral infection.

39 Keywords: Cellular response; PABPs; Toxoplasma infection; nuclear granules; host-parasite

40 interaction 


\section{Introduction}

Toxoplasma gondii is an obligate intracellular parasitic protozoan of the phylum

43 Apicomplexa, which includes clinically and agriculturally important pathogens, Plasmodium, the

44 organism responsible for malaria; Eimeria and Cryptosporidium, enteric pathogens; and

45 Neospora and Theileria, systemic veterinary pathogens. Having felines as its definitive host, the

46 sexual reproduction of $T$. gondii occurs in the feline intestinal epithelial lining. Un-sporulated

47 oocysts are passed in the fecal matter at which point the process of sporogony takes place outside

48 the definitive host and results in sporulated oocysts containing infective sporozoites (Tenter et al.

49 2000). Following ingestion by an intermediate host, which includes various warm-blooded

50 animals including humans, the sporozoites will lyse from the oocyst and will differentiate into the

51 tachyzoite form of the parasite. In its intermediate host cell, the rapidly growing tachyzoite form

52 reproduces asexually with a doubling time of about six to eight hours until the lysis of the host

53 cell. Approximately seven to ten days after infection of its intermediate host, tachyzoites will

54 begin to differentiate into the quiescent bradyzoite form, which forms tissue cysts mainly in

55 muscle and central nervous system tissue. Upon entering the digestive tract, ingested tissue cysts

56 will burst leading to release of the bradyzoites, which will subsequently infect the intestinal

57 epithelium and differentiate into the tachyzoite form of the parasite (Black and Boothroyd 2000).

58 It has been estimated that about one-third of the population are affected by $T$. gondii

59 infection; the infection of which could lead to the development of clinical toxoplasmosis. The

60 disease's prevalence is affected by several factors, including socioeconomic status of the area.

61 Most of cases in humans are the result of ingestion of oocysts in food or water, which has been

62 contaminated with cat feces, or ingestion of tissue cysts in raw or undercooked meat. Congenital

63 infection results when a woman becomes infected while she is pregnant and the infective

64 tachyzoites are transmitted through the placenta to the fetus. This results in the possibility of a 
65 variety of issues for the infected child, including blindness as well as the possibility of severe

66 neurological conditions including hydrocephalus, and may even result in spontaneous abortion.

67 In most patients, toxoplasmosis is asymptomatic (Montoya and Liesenfeld 2004). The tachyzoite

68 form of the parasite is repressed by the host's immune system, which causes differentiation of the

69 parasites into the slow-dividing and latent bradyzoite form (Blader et al. 2015). However, in

70 immunocompromised individuals, including those with HIV/AIDS or those undergoing

71 chemotherapeutic treatment, the disease can be life-threatening and usually results from

72 reactivation of latent infection. In these cases, patients can suffer from a variety of health issues

73 including chorioretinitis, encephalitis, acute respiratory failure or pneumonitis (Montoya and

74 Liesenfeld 2004). The treatment of $T$. gondii infection includes the combination of

75 pyrimethamine and sulfonamide; however, the treatment is not effective against tissue cysts and

76 has toxic side effects (Black and Boothroyd, 2000). A better understanding of host-parasite

77 interaction is required in the development of preventative treatments and therapies of chronic

78 toxoplasmosis.

Poly-adenosine binding proteins (PABPs) play an important role in eukaryotic cells'

80 response to infection and stress. PABPs are an abundant family of RNA binding proteins found in

81 eukaryotes, which bind to polyadenylated RNA with both strong specificity and affinity for the

82 poly (A) tail (Wigington et al. 2014). This class of proteins recognizes the 3' poly (A) tail, which

83 is added to mRNA precursors during 3'-end processing in the nucleus, and is essential for

84 stabilizing mRNA, for efficient export of mRNA to the cytosol, and for the initiation of

85 translation in the cytoplasm. Although PABPs do not display catalytic activity, they are

86 multifunctional scaffolds involved in all metabolic pathways of the mRNA (Eliseeva et al. 2013).

87 In eukaryotes there are cytoplasmic and nuclear PABPs, each with their respective roles. In

88 metazoans, multiple genes encode PABPs (Goss and Kleiman 2012). In humans, there are seven 
89 PABPs, including one nuclear termed PABPN1, four cytoplasmic PABPs which include 90 PABPC1, PABPC3, PABPC4 and PABPC4L, in addition to an embryonic protein called ePABP 91 (or PABPC1L), and an X chromosome encoded protein termed PABPC5 (Eliseeva et al. 2013).

92 The cytoplasmic PABPs bind the poly (A) tail of mRNA and interacts with the initiation factor 93 eIF4G, circularizing the mRNA for translational initiation. Therefore, PABPBS are considered a 94 marker for monitoring translation (Kühn and Wahle 2004). Under stressful conditions, PABPs can be relocalized to multiple and/or distinct sites

96 (Gray et al. 2015). For instance, following UV irradiation both PABPC1 and PABPC4 briefly 97 localize to cytosolic stress granules, and subsequently to the nucleus (Burgess et al. 2011). It was 98 postulated that their nuclear relocalization may have a role in destruction of the transcriptome 99 (Gray et al. 2015). In addition, the Rotavirus infection has been shown to cause displacement of 100 PABP to the nucleus (López et al. 2016). It is also possible that the relocalization of PABPs 101 reflects mRNA localization and turnover. Here we report an observation of the relocalization of 102 PABPs in human host cells upon T. gondii infection. Notably the aggregates of PABPs formed 103 upon infection are mainly found in the nucleus, which is different from the response found in an 104 exposure to a heat shock. The treatment of the infected monolayers with pyrimethamine inhibits 105 the multiplicity of the parasite, and reverses the relocalization of PABP aggregates. This active 106 interaction between the infected mammalian host cells and $T$. gondii appears to be different than 107 that caused by viral infection, further confirming that PABPs is an ideal protein marker to 108 decipher an innate protective response caused by infection.

\section{Materials and methods}




\section{Mammalian cell and parasite cultures}

113 Human fibroblasts (ATCC-SCRC-1041), were cultured using a media consisting of

114 Dulbecco's Modified Eagle Medium (Invitrogen, 12100046) supplemented with 10\% Cosmic Calf

115 Serum (HyClone, H3008704N), and 0.5x antibiotic-antimycotic reagent (Invitrogen, 15240-062). T.

116 gondii, RH$\Delta \mathrm{HX}$ strain (ATCC-50174), was maintained by infecting confluent human fibroblast

117 monolayers and culturing using a media, consisting of Minimum Essential Medium (Invitrogen,

118 61100061) supplemented with 1\% dialyzed fetal bovine serum (ThermoFisher Scientific, Hyclone,

119 SH3007903) and 0.5x antibiotic-antimycotic. The parasites were subcultured every 2-3 days

120 following lysis from the monolayers. Both human fibroblast and parasite cultures were maintained

121 in a $37^{\circ} \mathrm{C}$ incubator with $5 \% \mathrm{CO}_{2}$ until used.

123 Western blot analysis

124 The lysates were denatured in 1x SDS loading dye, and then boiled before resolving on a

$12510 \%$ SDS-PAGE. Resolved proteins were transferred to a nitrocellulose membrane, and blocked in $1265 \%(\mathrm{w} / \mathrm{v})$ non-fat skim milk in TBST for 1 hour. The blot was then incubated in PABPC3127 polyclonal antibody (PABPC3, 1: 1,000, ABM, Y058722) in 2\% (w/v) non-fat skim milk in TBST.

128 The blot was subsequently washed with TBST and then incubated in HRP-conjugated goat-anti129 rabbit antibody in $2 \%(\mathrm{w} / \mathrm{v})$ non-fat skim milk in TBST. The secondary antibody used for this study 130 was HRP-conjugated goat anti-rabbit (Rockland Immunochemicals Inc., \#611-1322) at a 1: 10,000 131 dilution. The blot was again washed with TBST and then incubated with Chemiluminsecent HRP 132 Substrate Kit (Millipore, \#WBKLS0500) and imaged using the FluorChem Q Imager (Alpha 133 Innotech) with AlphaView-FluorChem Q software.

\section{Treatment conditions}


136 Heat shock treatment was performed by incubating human fibroblast monolayers at $42^{\circ} \mathrm{C}$ in

$1375 \% \mathrm{CO}_{2}$ incubator for 2 hours. Treatments with pyrimethamine (Sigma-Aldrich, P7771) were 138 carried out 24 hours post-infection by adding pyrimethamine into the culturing media of infected 139 monolayers (1 $\mu \mathrm{M}$ final concentration).

141 Immunofluorescence assay

142 Human fibroblast monolayers were grown on coverslips $(22 \times 22 \mathrm{~mm})$. The coverslips with 143 infected and non-infected human fibroblast monolayers were washed with 1x PBS, fixed with 3\% 144 paraformaldehyde for 5 minutes, and then treated with $0.25 \%$ triton in 1 x PBS for 10 minutes. Non145 specific binding was prevented by incubating in 5\% BSA (Fisher Scientific) or 5\% equine serum 146 (HyClone, SH30074). An antiserum raised against human polyclonal PABPC3 (1:500, ABM, 147 Y058722), and FITC-conjugated goat anti-rabbit secondary antibody (1:1000, Rockland, 611-102148 122) were used. Hoechst 33342 (Invitrogen, H1399) was used for nuclear staining. The coverslips 149 were mounted onto microscope slides with Fluoromount-G (Electron Microscopy Sciences, 17984150 25), and viewed by fluorescence microscopy (Leica DMI 6000 fluorescence microscope with Leica 151 Application Suite Advanced Fluorescence Software). Approximately 100 independent fields of 152 view were taken, and the images were analyzed by CellProfiler 2.2.0 (Dao et al. 2016; Jones et al. 153 2008). Data were compiled into Microsoft Excel spreadsheets, then averages and standard errors 154 were calculated for each infection time point.

\section{Results and discussion}

158 In eukaryotes, PABP is known to be a component of stress granules (Anderson and 159 Kedersha 2008). To ensure that the PABP detection used in the study would allow for the 
160 monitoring of stress-induced granule formation, monolayers of human fibroblasts were subjected to

161 a heat shock treatment under conditions known to cause cellular stress responses, and analyzed by

162 immunofluorescence assay. The aggregations of PABPs were detected in both the cytoplasm and

163 nucleus of the host cells (Figure 1A). Following heat shock treatment, PABP-aggregates were

164 noticeably increased when compared to the control. To determine whether the increase was due to

165 the formation of PABP-containing mRNP granules or an increase in total PABPs, Western blot

166 analysis was performed using cell lysates prepared from the control and heat shocked human

167 fibroblast monolayers, and revealed by a polyclonal antibody raised against human PABPC3, which

168 has been shown to detect PABP homologs in different cell types (Marque et al. 2005). Here,

169 resolved protein bands were detected around 60 and $80 \mathrm{kDa}$, while the predicted molecular masses

170 of human PABPs without post-translational modification (UniProt Consortium) are $70.7 \mathrm{kDa}$

171 (PABPC1), $70.0 \mathrm{kDa}$ (PABPC3), $70.8 \mathrm{kDa}$ (PABPC4), $41.9 \mathrm{kDa}$ (PABPC4L), $32.7 \mathrm{kDa}$

172 (PABPN1), $68.4 \mathrm{kDa}(\mathrm{PABPC} 1 \mathrm{~L})$, and $43.3 \mathrm{kDa}$ (PABPC5). The discrepancy of sizes of revealed

173 proteins and predicted molecular mass is not surprising. Human PABPC1 has at least 14 post-

174 translational modifications, including the acetylation of lysine residues, the methylation of

175 glutamate, aspartate, lysine and arginine residues, and the dimethylation of lysine residues, found

176 throughout its functional domains (Brook et al. 2012). We observed no differences between the

177 intensities of both PABP revealed bands detected in the control and heat shock treatments (Figure

178 1B). It thus indicated that 2-hour heat shock treatment did not increase de novo PABP synthesis, but

179 induced formation of PABP-aggregates.

180

181 Heat-shock and parasite infection

182 Naturally at any given time, human cells could simultaneously be assaulted by multiple 183 cellular stresses (e.g. heat or cold shock, nutrient deprivation, and infection). We questioned how 
184 this stress-induced response would affect the host's ability to cope with an infection caused by $T$.

185 gondii. To address this, we compared the progression of $T$. gondii multiplication following an

186 infection of host cells with and without prior heat-shock treatments. As an intracellular obligate

187 parasite, $T$. gondii replicates within a parasitophorous vacuole by the mode of endodyogenous

188 replications. Herein to follow the progression of $T$. gondii infection, we assessed the number of

189 parasites per vacuole formed in infected host monolayers. With or without prior heat shock

190 treatment, the host cells allowed a similar T. gondii replication. Twenty-four hours post-infection,

$19135-40 \%$ of vacuoles carried 4 parasites per vacuole (Figure 1C). It thus indicated that the

192 intracellular multiplication of $T$. gondii was not affected by the host cells' stress response under the 193 conditions used.

\section{Toxoplasma infection and the host cell's stress response}

While assessing the host cell's responses to a heat shock and $T$. gondii infection, we

197 observed a significant increase in the formation of PABP-granules in the nucleus, referred to here as

198 nuclear PABP-granulation, in infected human fibroblasts at 24 hours post-infection, as compared to

199 uninfected host monolayers (Figure 2). To determine the effect of parasitic infection on the nuclear

200 PABP-granulation as a host cell's stress response, we performed a time-course analysis. We

201 monitored nuclear PABP-granulation at different intervals following an infection over a period of

20248 hours, or until T. gondii infection caused the host cells to lyse. Figure 3A shows representative

203 images of infected human fibroblasts. Hoechst staining was used to identify the boundaries of the

204 nucleus and to allow for the quantification of the fluorescence signals derived from the nuclear

205 PABP-granulation. Approximately 100 - 300 independent fibroblasts were analyzed and their

206 fluorescence signals revealed by a polyclonal PABPC3 antibody were recorded for each time point

207 as relative fluorescence units (RFU, Figure 3B). When an average fluorescence signal per nucleus 
208 was plotted as a function of time since infection, we found a linear relationship with the increase

209 intensity due to the nuclear granule formation with the rate of 0.31 hour $^{-1}$ (Figure 3B). To determine

210 whether the increase of PABP-fluorescence signals in the nucleus was the result of more PABP

211 accumulation in each granule, or more granules per nuclei, we further performed a morphometric

212 analysis using CellProfiler software. The number and size of nuclear granules were analyzed for

213 each time point. On average, both number and size of nuclear granules increased following $T$.

214 gondii infection (Figure 3C, Supplementary Figures S1 and S2). While the average size of nuclear

215 PABP granules was unchanged at the end of the first hour post-infection, the number of granules

216 was slightly increased. At 7 hours post-infection, both average size and average number of nuclear

217 PABP granules were increased $\sim 20 \%$. At 24 hours post-infection, the average number of nuclear

218 PABP granules was at its peak of $\sim 15$ granules/nucleus, while the average size was $\sim 13 \mu \mathrm{m}^{2}$. At the

219 end of 48 hours post-infection, the average size of the granules reached its peak of $\sim 15 \mu \mathrm{m}^{2}$ (Figure

$2203 \mathrm{C}$, hatched bars) At this time point, some human fibroblasts were infected with freshly released $T$.

221 gondii (secondary infection). It is possible that the number of granules has reached its maximal

222 level, and that the secondary infection caused the granule number to increase further (Figure $3 \mathrm{C}$,

223 dark bars). A gradual increase in the quantity of PABP-granules during the few hours following

224 infection could indicate that the infection elicits a defense mechanism, in which mRNAs are

225 accumulated in the nuclear compartment. The longer the parasites proliferate inside the host cell,

226 more translational materials (mRNAs and various RNA binding proteins) are sequestered into

227 larger mRNP granules. As infection progressed, so did the granulation of PABP in the host nucleus.

228 However, it remains to be investigated whether a same phenomenon takes place in different cell

229 types, including muscle, kidney or liver cells, and/or with a higher parasite load. In human

230 fibroblasts used here, we postulate that these nuclear granules are part of host-parasite interaction,

231 and that is similar to cytoplasmic stress granules. To fight infection, the translational machinery and 
232 mRNA are repressed, until the stress is relieved. These observations paralleled findings with

233 Rotavirus, which also causes displacement of host PABP to the nucleus upon infection (López et al.

234 2016). However, while Rotavirus infection inhibits the formation of stress granules in the host

235 (Montero et al. 2007), T. gondii infection appears to be inducing the formation of nuclear granules

236 in a time-dependent manner.

\section{Relocalization of PABPs following T. gondii infection}

Since the accumulation of PABPs in the nucleus of human fibroblasts was observed to be

240 directly correlated with the progression of $T$. gondii infection, we questioned whether the removal

241 of the parasites may reverse this relocalization of PABP. Cultures of human fibroblasts were

242 infected with $T$. gondii and allowed to grow for 24 hours prior to the addition of pyrimethamine, an 243 antiparasitic agent. Pyrimethamine is a potent dihydrofolate reductase inhibitor with an $\mathrm{IC}_{50}$ of 0.25

244 to $1.5 \mu \mathrm{M}$ (Ben-Harari et al. 2017). Pyrimethamine treatments of $T$. gondii-infected monolayers 245 were analyzed at different intervals (12-48 hours) for the accumulation of nuclear PABP-granules.

246 A decrease in nuclear PABP granulation was observed after 12 hours of pyrimethamine treatment 247 (Figure 4). The results confirmed that the infection and growth of T. gondii cause a stress-induced 248 response in the host leading to the formation of nuclear PABP-containing granules.

249 During the invasion of the host cell by Apicomplexan parasites, specialized secretory 250 organelles, including micronemes, rhoptries, and dense granules, release their protein contents into 251 the host cell. These proteins are speculated to interact with those of the host cell leading the 252 alteration of host cell's biology (Gilbert et al. 2006). For example, members of the dense granule 253 protein family called GRA proteins of $T$. gondii could elicit changes in the host's gene expression. 254 Once successfully invading the host cell, $T$. gondii replicates within a parasitophorous vacuole and 255 continues to secrete their proteins into the parasitophorous vacuole and then into the host cell. Two 
256 members of GRA proteins, namely GRA16 and GRA24, are able to cross the membrane of the 257 parasitophorous vacuole and migrate to the host nucleus (Bougdour et al. 2014). The interaction of 258 these proteins with host cell proteins affects the regulation of host cell's gene expression through 259 disrupting certain signaling pathways, which may be a potential cause for this nuclear PABP260 granulation. Upon infection, the parasite also secretes from its rhoptries TgPP2C-hn, a protein 261 phosphatase, into its host. This protein then traverses to the nucleus where its target molecules are 262 not yet known. The secretion of this phosphatase, a common enzymatic effector of cell signaling 263 pathways, is another example of a way that the parasite interacts with and potentially modulates its 264 host (Gilbert et al. 2006). As a nuclear directed protein, this phosphatase may provide another 265 possible explanation for the infection-induced nuclear granulation seen in this study. To further 266 elucidate a mechanism by which $T$. gondii manipulates the host's cellular response, one could 267 utilize a chemical compound known to specifically inhibit invasion or egress of the parasite from 268 the host cells. For example, cytochalasin D, an inhibitor of actin polymerization, can be used in 269 causing T. gondii to secrete its rhoptry factor, but inhibiting parasite invasion (Caldas et al. 2013).

270 The experiment would address whether the host cell's PABP granulation is directly correlated with 271 the rhoptry secretion and/or T. gondii invasion.

274 Conclusion

275 This study describes an observation of a host-parasite interaction, where $T$. gondii infection 276 causes granulation of the RNA-binding protein, PABPs, in the host cell. This stress response differs 277 from a heat shock treatment that generally causes both cytoplasmic and nuclear granulation of 278 PABPs in eukaryotes. T. gondii infection specifically led to pronounced nuclear PABP granulation, 279 which increased over the period of infection. In addition, the removal of the parasites by 
280 chemotherapeutic agent reversed the process of nuclear granulation, confirming that the infection 281 and growth of the parasites was causing PABP to aggregate in the nucleus in a time-dependent 282 manner. Prospective work would be to study the molecular pathway leading to this stress-induced 283 response to further understand the dynamic interaction between host and pathogen. The better 284 understanding of how $T$. gondii manipulates the host's response will help elucidate host-parasite 285 interaction, and open an avenue to disrupt and prevent the parasite infection.

\section{Acknowledgements}

289 This work was supported by the Natural Sciences and Engineering Research Council of Canada 290 (NSERC, Canada, S.A.). MR and YA are the recipients of NSERC-USRA. SR is the recipient of 291 Canada Graduate Scholarships-Master's (CGS-M) Program, and Ontario Graduate Scholarship in 292 Science and Technology (OGS-ST).

\section{Conflicts of interest}

295 The authors declare no competing financial interest.

\section{Author contributions}

299 KF, MR, and YA performed experiments, and analyzed data. SA conceived and coordinated the 300 study. SR and SA conceptualized and designed the experiments. KF, MR and SA wrote the paper. 301 All authors analyzed the results and approved the final version of the manuscript. 


\section{References}

Anderson, P., and Kedersha, N. 2008. Stress granules: the Tao of RNA triage. Trends in Biochemical Sciences, 33(3), 141-150. doi: 10.1016/j.tibs.2007.12.003.

Ben-Harari, R.R., Goodwin, E., and Casoy, J. 2017. Adverse Event Profile of Pyrimethamine-Based Therapy in Toxoplasmosis: A Systematic Review. Drugs R D. 17(4):523-544. doi: $10.1007 / \mathrm{s} 40268-017-0206-8$.

Black, M.W., and Boothroyd J.C. 2000. Lytic Cycle of Toxoplasma gondii. Microbiology and Molecular Biology Reviews, 64(3), 607-623. doi:10.1128/mmbr.64.3.607-623.2000.

Blader, I.J., Coleman, B.I., Chen, C., and Gubbels, M. 2015. Lytic Cycle of Toxoplasma gondii: 15 Years Later. Annual Review of Microbiology, 69(1), 463-485. doi:10.1146/annurev-micro091014-104100.

Bougdour, A., Tardieux, I., Hakimi, M. 2014. Toxoplasma exports dense granule proteins beyond the vacuole to the host cell nucleus and rewires the host genome expression. Cellular Microbiology, 16(3), 334-343. doi:10.1111/cmi.12255.

322 Brook, M., McCracken, L., Reddington, J.P., Lu, Z.L., Morrice, N.A., and Gray, N.K. 2012. The multifunctional poly(A)-binding protein (PABP) 1 is subject to extensive dynamic posttranslational modification, which molecular modelling suggests plays an important role in coordinating its activities. Biochem Journal, 441(3):803-812. doi: 10.1042/BJ20111474. PubMed PMID: 22004688. 
327 Burgess, H.M., Richardson, W.A., Anderson, R.C., Salaun, C., Graham, S.V., and Gray, N.K. 2011. 328 Nuclear relocalization of cytoplasmic poly(A)-binding proteins PABP1 and PABP4 in response to UV irradiation reveals mRNA-dependent export of metazoan PABPs. Journal of Cell Science, 124(19), 3344-3355. doi:10.1242/jcs.087692.

Caldas, L.A., Seabra, S.H., Attias, M., and de Souza, W. 2013. The effect of kinase, actin, myosin 332 and dynamin inhibitors on host cell egress by Toxoplasma gondii. Parasitol Int. 62(5):475-82. doi: 10.1016/j.parint.2013.04.006.

Dao, D., Fraser, A.N., Hung, J., Ljosa, V., Singh, S., and Carpenter, A.E. 2016. CellProfiler 335 Analyst: interactive data exploration, analysis, and classification of large biological image sets. doi:10.1101/057976.

Eliseeva, I.A., Lyabin, D.N., and Ovchinnikov, L.P. 2013. Poly(A)-binding proteins: Structure, domain organization, and activity regulation. Biochemistry (Moscow), 78(13), 1377-1391. doi:10.1134/s0006297913130014.

340 Gilbert, L.A., Ravindran, S., Turetzky, J.M., Boothroyd, J.C., and Bradley, P.J. 2006. Toxoplasma gondii Targets a Protein Phosphatase 2C to the Nuclei of Infected Host Cells. Eukaryotic Cell,

Goss, D.J., and Kleiman, F.E. 2012. Poly(A) binding proteins: are they all created equal? Wiley 6(1), 73-83. doi:10.1128/ec.00309-06. Interdisciplinary Reviews: RNA, 4(2), 167-179. doi:10.1002/wrna.1151

Gray, N.K., Hrabalkova, L., Scanlon, J.P., and Smith, R.W. 2015. Poly(A)-binding proteins and mRNA localization: who rules the roost? Biochemical Society Transactions, 43(6), 12771284. doi:10.1042/bst20150171. 
348 Jones, T.R., Kang, I., Wheeler, D.B., Lindquist, R.A., Papallo, A., Sabatini, D.M., and Carpenter, A.E. 2008. CellProfiler Analyst: data exploration and analysis software for complex imagebased screens. BMC Bioinformatics, 9(1), 482. doi:10.1186/1471-2105-9-482.

351

352

353

354

Kühn, U., and Wahle, E. 2004. Structure and function of poly(A) binding proteins. Biochimica et Biophysica Acta (BBA) - Gene Structure and Expression, 1678(2-3), 67-84. doi:10.1016/j.bbaexp.2004.03.008.

López, S., Oceguera, A., and Sandoval-Jaime, C. 2016. Stress Response and Translation Control in Rotavirus Infection. Viruses, 8(6), 162. doi:10.3390/v8060162

Marques, A.C., Dupanloup, I., Vinckenbosch, N., Reymond, A., and Kaessmann, H. 2005. Emergence of young human genes after a burst of retroposition in primates. PLoS Biol., 3(11):e357.

Montero, H., Rojas, M., Arias, C.F., Lopez, S. 2007. Rotavirus Infection Induces the Phosphorylation of eIF2 but Prevents the Formation of Stress Granules. Journal of Virology, 82(3), 1496-1504. doi:10.1128/jvi.01779-07.

Montoya, J., and Liesenfeld, O. 2004. Toxoplasmosis. The Lancet, 363(9425), 1965-1976. doi:10.1016/s0140-6736(04)16412-x.

Tenter, A.M., Heckeroth, A.R., and Weiss, L.M. 2000. Toxoplasma gondii: from animals to humans. International Journal for Parasitology, 30(12-13), 1217-1258. doi:10.1016/s00207519(00)00124-7.

UniProt Consortium. 2017. UniProt: the universal protein knowledgebase. Nucleic Acids Research, 45(D1):D158-D169. doi: 10.1093/nar/gkw1099.

van der Ven, A.J., Schoondermark-van de Ven, E.M., Camps, W., Melchers, W.J., Koopmans, P.P., van der Meer, J.W., and Galama, J.M. 1996. Anti-toxoplasma effect of pyrimethamine, 
371 trimethoprim and sulphonamides alone and in combination: implications for therapy. Journal 372 of Antimicrobial Chemotherapy, 38(1):75-80.

373 Wehner, K.A., Schütz, S., and Sarnow, P. 2010. OGFOD1, a novel modulator of eukaryotic 374 translation initiation factor 2alpha phosphorylation and the cellular response to stress. 375 Molecular and Cell Biology, 30(8):2006-16. doi: 10.1128/MCB.01350-09.

376 Wigington, C.P., Williams, K.R., Meers, M.P., Bassell, G.J., and Corbett, A.H. 2014. Poly(A) 377 RNA-binding proteins and polyadenosine RNA: new members and novel functions. Wiley 378 Interdisciplinary Reviews: RNA. 5:601-622 doi:10.1002/wrna.1233. 
Figure legends

382 Figure 1 Formation of stress-induced PABP-mRNP granules. (A) Following a two-hour

383 heat shock treatment at $42^{\circ} \mathrm{C}$, PABP-containing aggregates were detected throughout the nucleus

384 and the cytosol of human fibroblasts. Micrometer bars represent $10 \mu \mathrm{m}$. (B) Western blot analysis

385 revealed similar level of PABPs in human fibroblasts (HF) with and without a heat shock treatment.

386 Protein lysates were resolved on 10\% SDS-PAGE, transferred to a nitrocellulose membrane, and

387 probed with PABPC3-antisera. The sizes of protein markers are indicated, and an arrow identifies

388 the revealed PABP at $\sim 80 \mathrm{kDa}$. (C) Replication of T. gondii in human fibroblasts was observed at

38924 hours post-infection of the human fibroblasts with and without a heat shock treatment. Number

390 of parasites per vacuole were counted from $\sim 100$ independent vacuoles, and their average values

391 were plotted. The experiments were done in replicates, and similar results were detected.

392

393 Figure 2 Human fibroblasts in response to $\boldsymbol{T}$. gondii Infection. Representative images of $T$.

394 gondii infected and uninfected human fibroblasts are shown. PABPC3-antisera reveal the locations

395 of PABPs in the nucleus and cytosol of $T$. gondii as well as the nucleus of the human fibroblasts.

396 Arrow indicates infected host cell nucleus and arrow-head indicates uninfected host cell nucleus

397 Micrometer bars represent $10 \mu \mathrm{m}$.

399 Figure 3 Time-course analysis of PABP-containing granules following $T$. gondii infection.

400 (A) Representative images of $T$. gondii infected human fibroblasts from different time points post401 infection. (B) Independent images were taken with a constant exposure time, and resolution to 402 allow for the measurement of fluorescent intensity of PABP-aggregates formed within the nucleus 403 of the fibroblasts. Average fluorescence intensity was plotted as a function of post-infection time (1 $404-48$ hours). Due to low fluorescence signals detected in uninfected fibroblasts (control), the 
405 exposure time and resolution were altered for visualization; the fluorescence intensity obtained 406 from the control (time $=0$ hour) was thus not included in the plot. (C) Morphometric analysis was 407 performed to obtain the number and size of infection-induced PABP granules. The average size 408 (solid bars) and the average number (hatched bars) were obtained from independent $100-300$ 409 nucleus were plotted as a function of post-infection time (see supplementary Figures S1 and S2 for 410 the data distribution for the number and size of granules).

411

412 Figure 4 Pyrimethamine treatment of $\boldsymbol{T}$. gondii-infected fibroblasts. $T$. gondii was allowed 413 to infect human fibroblasts for 24 hours prior to the treatment with pyrimethamine $(1 \mu \mathrm{M})$. 414 Representative images are shown. It was observed that the presence of pyrimethamine diminished 415 the parasite growth and decreased the formation of nuclear PABP granules. 


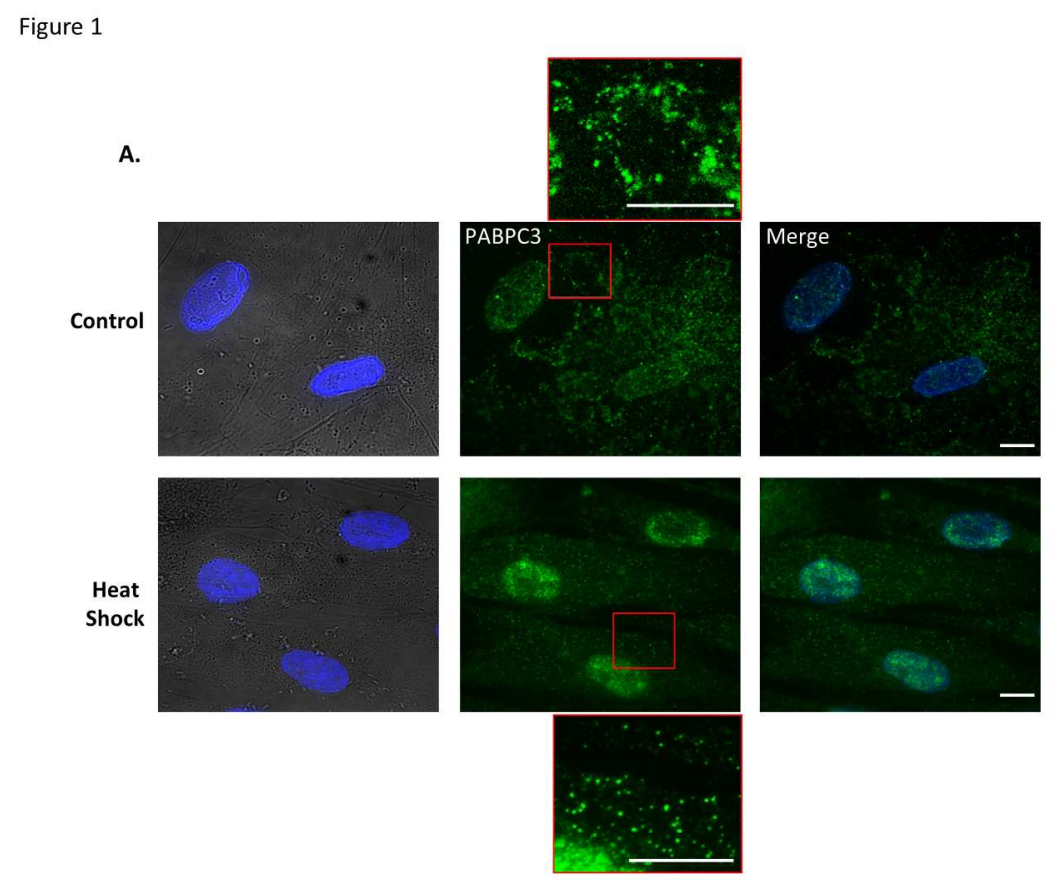

B.

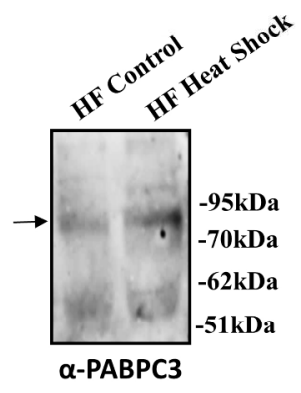

c.

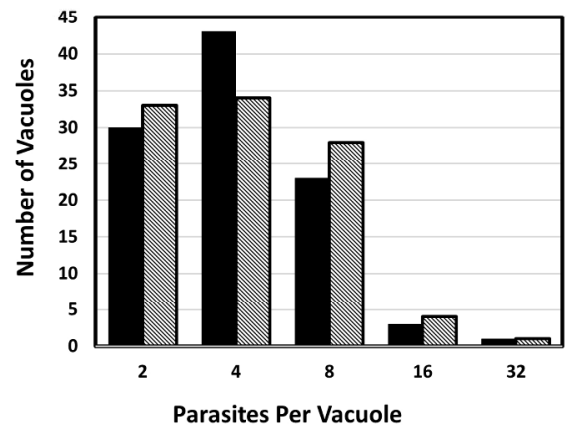

Figure 1 Formation of stress-induced PABP-mRNP granules. (A) Following a two-hour heat shock treatment at $42^{\circ} \mathrm{C}$, PABP-containing aggregates were detected throughout the nucleus and the cytosol of human fibroblasts. Micrometer bars represent $10 \mu \mathrm{m}$. (B) Western blot analysis revealed similar level of PABPs in human fibroblasts (HF) with and without a heat shock treatment. Protein lysates were resolved on $10 \%$ SDS-PAGE, transferred to a nitrocellulose membrane, and probed with PABPC3-antisera. The sizes of protein markers are indicated, and an arrow identifies the revealed PABP at $\sim 80 \mathrm{kDa}$. (C) Replication of T. gondii in human fibroblasts was observed at 24 hours post-infection of the human fibroblasts with and without a heat shock treatment. Number of parasites per vacuole were counted from $\sim 100$ independent vacuoles, and their average values were plotted. The experiments were done in replicates, and similar results were detected.

$$
190 \times 254 \mathrm{~mm}(300 \times 300 \mathrm{DPI})
$$


Figure 2
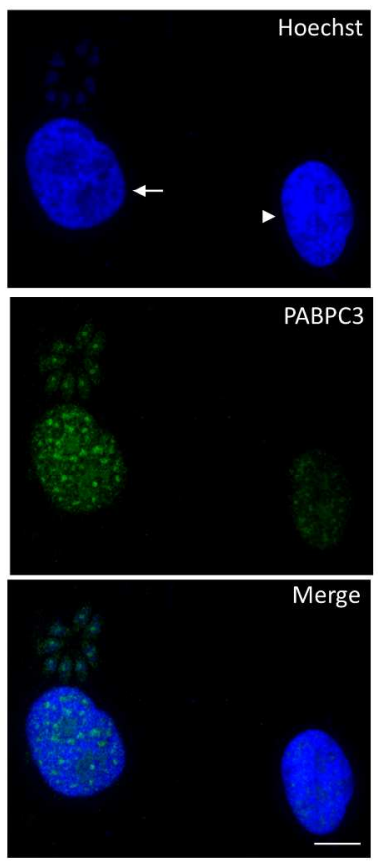

Figure 2 Human fibroblasts in response to T. gondii Infection. Representative images of T. gondii infected and uninfected human fibroblasts are shown. PABPC3-antisera reveal the locations of PABPs in the nucleus and cytosol of $T$. gondii as well as the nucleus of the human fibroblasts. Arrow indicates infected host cell nucleus and arrow-head indicates uninfected host cell nucleus Micrometer bars represent $10 \mu \mathrm{m}$.

$$
190 \times 254 \mathrm{~mm}(300 \times 300 \mathrm{DPI})
$$


Figure 3

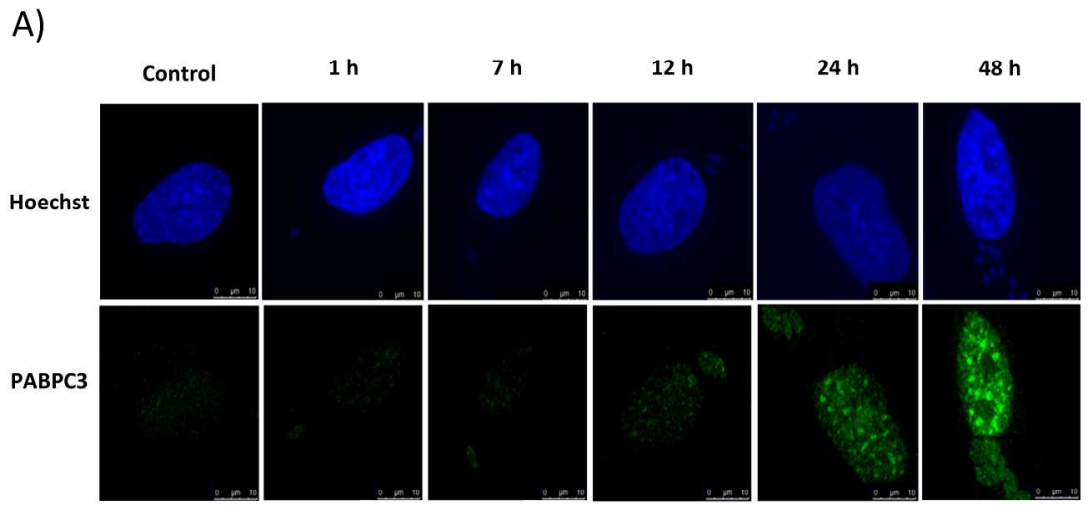

B)

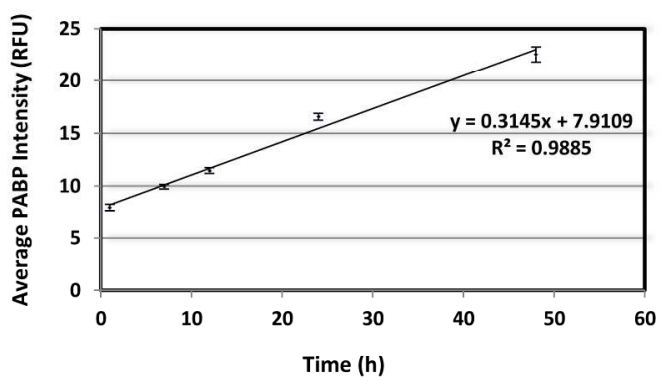

C)

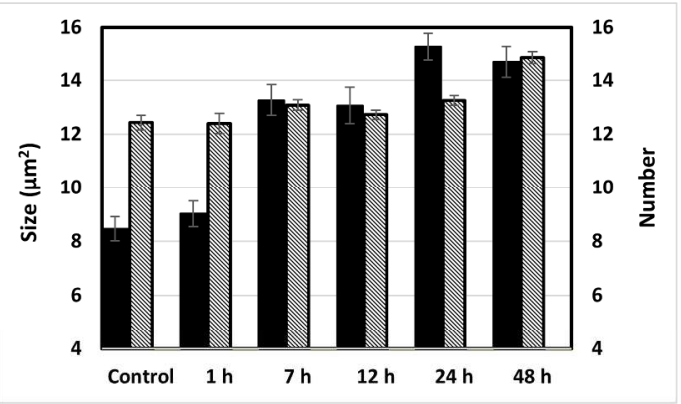

Figure 3 Time-course analysis of PABP-containing granules following T. gondii infection. (A) Representative images of $T$. gondii infected human fibroblasts from different time points post-infection. (B) Independent images were taken with a constant exposure time, and resolution to allow for the measurement of fluorescent intensity of PABP-aggregates formed within the nucleus of the fibroblasts. Average fluorescence intensity was plotted as a function of post-infection time (1 - 48 hours). Due to low fluorescence signals detected in uninfected fibroblasts (control), the exposure time and resolution were altered for visualization; the fluorescence intensity obtained from the control (time $=0$ hour) was thus not included in the plot. (C) Morphometric analysis was performed to obtain the number and size of infection-induced PABP granules.

The average size (solid bars) and the average number (hatched bars) were obtained from $100-300$ independent nucleus were plotted as a function of post-infection time (see supplementary Figures S1 and S2 for the data distribution for the number and size of granules). 
Figure 4

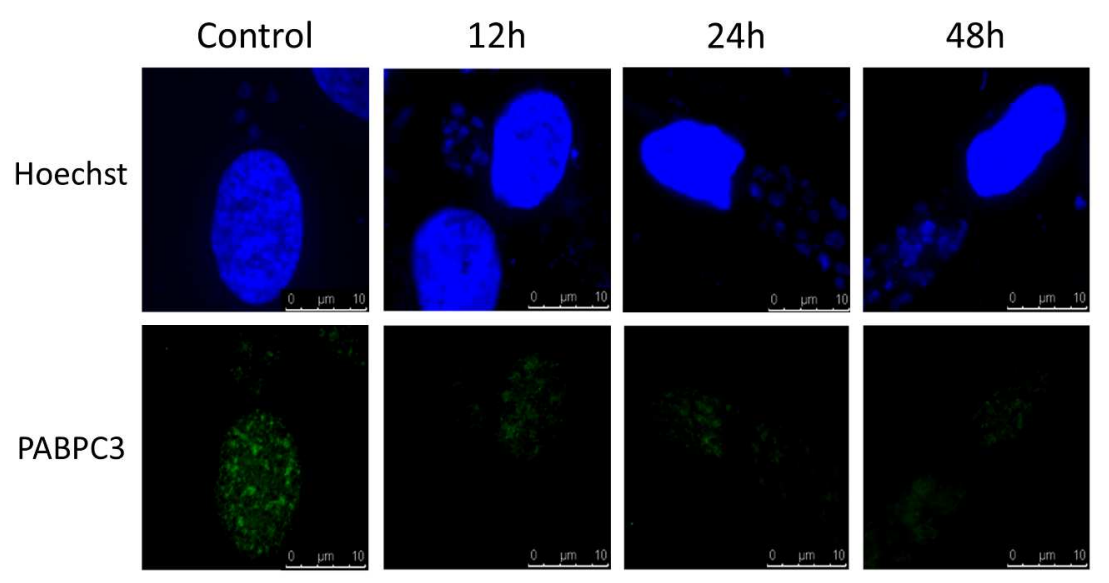

Figure 4 Pyrimethamine treatment of $T$. gondii-infected fibroblasts. $T$. gondii was allowed to infect human fibroblasts for 24 hours prior to the treatment with pyrimethamine $(1 \mu \mathrm{M})$. Representative images are shown. It was observed that the presence of pyrimethamine diminished the parasite growth and decreased the formation of nuclear PABP granules.

$$
190 \times 254 \mathrm{~mm}(300 \times 300 \text { DPI })
$$

